\title{
Dynamic properties of a sporadic sodium layer revealed by observations over Zhongshan, Antarctica: A case study
} 西

Xiangcai Chen ${ }^{1 *}$, Wentao Huang ${ }^{*}$, Chao Ban ${ }^{2}$, Michael J. Kosch ${ }^{3,4,5}$, Damian J. Murphy ${ }^{6}$, Zejun $\mathrm{Hu}^{1}$, Jianjun Liu ${ }^{1}$, Fang $\mathrm{He}^{1}$, Rui Wang ${ }^{1}$, Huigen Yang ${ }^{1}$, and Hongqiao $\mathrm{Hu}^{1}$

${ }^{1}$ MNR Key Laboratory for Polar Science, Polar Research Institute of China, Shanghai, China

${ }^{2}$ Key Laboratory of Middle Atmosphere and Global Environment Observation, Institute of Atmospheric Physics, Chinese Academy of Science, Beijing, China

${ }^{3}$ Department of Physics, Lancaster University, Lancaster, United Kingdom

${ }^{4}$ South African National Space Agency (SANSA), Hermanus, South Africa

${ }^{5}$ Department of Physics and Astronomy, University of the Western Cape, Bellville, South Africa

${ }^{6}$ Antarctic Climate Program, Australian Antarctic Division, Department of Agriculture, Water and the

Environment, Kingston, Tasmania, Australia

*Corresponding authors:

Xiangcai Chen (chenxiangcai@pric.org.cn) and Wentao Huang (huangwentao@pric.org.cn) 8

\section{Key points:}

20 1. Separation of temporal/spatial variations in SSLs is observed by a three-directional lidar system at Zhongshan, Antarctica.

2 . The formation and perturbance of SSLs are associated with a sporadic E layer at the same height.

3 . The formed SSLs are advected by the background wind. 


\section{Abstract}

26 A Na Doppler lidar system with three-directional measurements of sodium density, the atmospheric wind field, and the temperature was established at Zhongshan $\left(69.4^{\circ} \mathrm{S}, 76.4^{\circ} \mathrm{E}\right)$, Antarctica. On 14 November 2019, a sporadic sodium layer (SSL) was observed at altitude ranges of $93-103 \mathrm{~km}$. The temporal/spatial sodium density variations of this SSL are associated with a strong sporadic E (Es) layer at nearly the same height, which is modulated by the convective electric field. By considering the structures and the time lags of the SSL's growth at three positions, the SSL appears to have a horizontal advection in an approximately westward direction with a velocity of the order of $80 \mathrm{~m} / \mathrm{s}$. This is consistent with the zonal wind velocity derived from the lidar system itself. The temporal/spatial sodium density variations strongly indicate that the formation and perturbance of SSLs are related to the evolution of Es layers due to varied electric fields and atmospheric gravity waves, while it is advected by the horizontal wind.

\section{Plain Language Summary}

To be composed of metallic ions, the sporadic E layer (Es) could be formed, modified, or transported by the action of magnetospheric electric fields in the high latitude ionosphere. It has been widely proposed that the Es layer plays an important role in the formation of the sporadic sodium layers (SSL), but their detailed dynamic process and evolution studies of the Es/SSLs are still lacking. A three-frequency Sodium ( $\mathrm{Na}$ ) resonance fluorescence Doppler lidar has been recently deployed by the Polar Research Institute of China, which could measure the sodium density, temperature, and wind profiles simultaneously in three directions. To clarify the dynamic properties of Es/SSL, we have performed an event observation at Zhongshan Station $\left(69.4^{\circ} \mathrm{S}, 76.4^{\circ} \mathrm{E}\right)$, Antarctica, which includes sodium density profiles and wind velocity measured by multidirectional lidar system, Es layer detected by the collocated Digisonde radar, F region ion velocity, i.e., electric field, derived by SuperDARN HF radar, as well as gravity wave perturbation determined from Davis medium frequency radar. 


\section{Introduction}

Since the discovery of a sporadic sodium layer (SSL) that superposed on normal sodium layer in the mesosphere/lower thermosphere (MLT) [Clemesha et al., 1978], numerous SSL events have been reported [cf. Clemesha, 1995; Qiu et al., 2016, and references therein]. The SSLs, with the rapid growth of the sodium atom density over a narrow height range [e.g., Clemesha, 1995; Tsuda et al., 2011], usually show an extended life span from a few tens of minutes to several hours [e.g., Batista et al., 1991; Cox and Plane, 1998]. One of the interests in SSL studies is thus exploring the generation mechanism for SSLs and understanding the controlling factors of their temporal and spatial variations.

Ionospheric sporadic E (Es) layers have been widely accepted as a good candidate for the source of sodium atoms in SSLs [e.g., Cox and Plane, 1998; Kirkwood and Zahn, 1991; Takahashi et al., 2015]. Some good correlations between the occurrence of SSLs and Es layers have also been reported [cf. Croskey et al., 2006; Dou et al., 2009; 2010; Heinselman et al., 1998]. Rocket-bome mass spectrometric measurements have demonstrated that the Es layers consist of metal ions [Kopp, 1997; Grebowsky and Aikin, 2002], such as $\mathrm{Fe}^{+}, \mathrm{Mg}^{+}, \mathrm{Na}^{+}$, etc., and can be vertically driven by the neutral wind and/or the electric field [Kirkwood and Zahn, 1991]. When horizontal and vertical convergence of ions occurred under some conditions [e.g., MacDougall and Jayachandran, 2005], the ES layers could descend/ascend in altitude, such as due to the atmospheric tide and/or gravity waves [e.g., MacDougall et al., 2000]. As the atmospheric density increases exponentially with descending altitude, the rate of ion neutralization increases rapidly at lower heights [Collins et al., 2002]; an SSL could thus form via this rapid ion neutralization [Cox and Plane, 1998].

The other candidates for the source of sodium atoms could be direct meteor deposition, and/or release from aerosol particles, as well as redistribution of existing atoms [e.g., Batista et al., 1991; Clemesha, 1995; Clemesha et al., 1978]. However, direct meteor deposition requires a meteor shower with a large mass, and aerosol particle release requires a background temperature increase [Qiu et al., 2015; 2018]. The redistribution of existing atoms is another good explanation for SSLs. Using a steerable sodium lidar system at Winkfield $\left(51.4^{\circ} \mathrm{N}, 0.7^{\circ} \mathrm{W}\right)$ that pointed sequentially in three different directions (at zenith angles of $30^{\circ}$ ), Thomas et al. [1977] first observed clear evidence of a horizontal variation of the sodium concentration near the peak of the normal layer. Based on steerable lidar system observations at São José dos Campos $\left(23^{\circ} \mathrm{S}, 45^{\circ} \mathrm{W}\right)$, Batista et al., [1991] systematically investigated sodium density 
variations in twelve SSL events at three horizontal positions. There was no case where the horizontal advent of SSLs occurred simultaneously at all three positions; time lags of each SSL among the different positions were always observed. The inferred horizontal velocities from the time lags of SSLs were mostly less than $100 \mathrm{~m} / \mathrm{s}$, except for one case observed by Clemesha et al. [1980] with a velocity of the order of $200 \mathrm{~m} / \mathrm{s}$. However, their hypothesis of horizontal advection was not confirmed by background wind observations.

In a recent study, an SSL event detected with a five-directional lidar system was reported by Tsuda et al. [2015]. By utilizing the SSL onset time differences recorded at the five positions, the horizontal velocity of an SSL was derived and compared with the background wind velocity from the collocated meteor radar and European Incoherent Scatter radar; both velocities were consistent. Moreover, the amount of the sodium atom increase at the five positions was mostly equal, which strongly indicates that the observed SSL was just advected by the background wind. Nevertheless, the ending time of the SSL event was not observed by Tsuda et al. [2015] due to the sky becoming cloudy.

Although a causal link between the Es layer and SSL has been observed and reported [e.g., Heinselman et al., 1998; Kirkwood and Collis, 1989; Kirkwood and Zahn, 1991], how the temporal and spatial variations of SSL relate to the evolution of the Es layers and the horizontal advection by background wind is still unclear, comprehensive evaluation of the sodium atom production process in SSL and its related temporal and spatial variations with the Es layer and background wind is still an important question.

Since February 2019, a three-frequency sodium resonance fluorescence Doppler lidar system has been operating at Zhongshan Station (ZHS, $\left.69.4^{\circ} \mathrm{S}, 76.4^{\circ} \mathrm{E}\right)$, Antarctica. With three-directional beams toward the zenith, $30^{\circ}$ off-zenith to south and west, this system can obtain sodium density, line-of-sight wind, and temperature at three positions simultaneously. In this paper, an SSL event on 14 November 2019 that was detected by this lidar system is analyzed. We have performed detailed observations, including the ES layer by a colocated DPS-digisnode, electric field by the Super Dual Auroral Radar Network (SuperDARN), as well as wind velocity by the lidar system itself, to clarify the temporal and spatial variations of SSL. In addition, the $1.94 \mathrm{MHz}$ medium frequency (MF) radar [e.g., Reid et al., 2018] data from Davis station $\left(68.6^{\circ} \mathrm{S}, 78.0^{\circ} \mathrm{E}\right)$, which lies approximately $116 \mathrm{~km}$ northeast of ZHS, is used to assess the gravity wave activity below the SSL when it occurs. All these observations are 
combined to better understand the rapid sodium atom production process and its related dynamic properties in the MLT region. In section 2, we will briefly describe instruments and data sets. The observational results are presented in section 3, while possible generation processes and mechanisms of SSLs are discussed and proposed in section 4 . This paper finally ends with a summary in section 5 .

\section{Instruments and data sets}

\subsection{The sodium lidar}

The three-frequency Sodium (Na) resonance fluorescence Doppler lidar at ZHS mainly consists of the lidar transmitter, receiver, data acquisition, and system control modules. The lidar laser pulses are produced by a 4-stage pulsed dye amplifier seeded with a state-of-the-art $589 \mathrm{~nm}$ frequency-doubled solid-state diode laser. Required by the classic three-frequency probing technique [Chu and Papen, 2005, and reference therein], the seed laser is locked to one of the $\mathrm{Na}_{2 \mathrm{a}}$ Doppler-free saturation absorption features within $\pm 2 \mathrm{MHz}$, and then sequentially shifted $\pm 630 \mathrm{MHz}$ by a free-space acoustic-optical modulator. Three $82-\mathrm{cm}$ diameter microcrystal-glass substrate telescopes of the parabolic reflecting surface, i.e. the zenith, $30^{\circ}$ off-zenith to the south, and $30^{\circ}$ off-zenith to the west, are used to receive returning lidar photons in three directions for wind field measurement, respectively. While an ultra-narrow Na Faraday filter is implemented to suppress the solar background during daytime for continuous diurnal operation. With transmitted laser power of about $0.55 \mathrm{~W}$ in each direction, raw photons returned from the $\mathrm{Na}$ layer and collected by the receiving telescopes were integrated for $15 \mathrm{~s}$ with a $45 \mathrm{~m}$ range resolution. The uncertainties in temperature and vector wind measurements induced by photon noise, around the peak of Na layer $(\sim 90 \mathrm{~km})$ with 1 hour and $0.5 \mathrm{~km}$ resolutions, can achieve less than $\pm 0.3 \mathrm{~K}$ and $\pm 1.6 \mathrm{~m} / \mathrm{s}$ for nighttime, and $\pm 1.0 \mathrm{~K}$ and $\pm 2.8 \mathrm{~m} / \mathrm{s}$ for daytime, respectively.

\subsection{The Ionosonde radar (DPS-4D)}

The Digisonde Portable Sounder (DPS-4D) operated at ZHS can be used to monitor the overhead ionosphere. It uses one simple crossed delta antenna for transmission, and four crossed magnetic dipole antennas for reception. Using six digitally synthesized off-vertical reception beams in addition to the vertical beam, the DPS-4 digisonde can operate in the multi-beam sounding mode [Reinisch et al., 
2009]. For each frequency-range pixel, the beam with the maximum amplitude is selected, and the amplitude and beam numbers are recorded in the output data [Reinisch et al., 2008]. Currently, the digisonde operates at $0.05 \mathrm{MHz}$ frequency step from 0.5 to $9.5 \mathrm{MHz}$ and a spatial resolution of $2.5 \mathrm{~km}$ from 80 to $640 \mathrm{~km}$ (virtual height), while the ionograms are recorded at a time interval of 7.5 mins. By manually scaling the ionograms via SAO software, the ionospheric characteristics parameters such as Es critical frequency ( $f \mathrm{oEs}$ ) and virtual height (h'Es) can be obtained.

\subsection{The SuperDARN radar}

The fields of view of SuperDARN radar cover the majority of the northern and southern hemispheres in the polar region. Utilizing the HF radio wave refracted to achieve orthogonality with the Earth's magnetic field in the E- and F-region ionosphere, the field-aligned irregularities at $\sim 10 \mathrm{~m}$ Bragg scale has maximum backscatter power obtained [Milan et al., 1997]. From the received signal-to-noise ratios the ionospheric plasma Doppler line-of-sight velocity, the Doppler spectral power, and the Doppler spectral width can be derived [Greenwald et al., 1995]. Based on all the available velocity data and merging it from pairs of radars within common-volume areas [Ruohoniemi and Baker, 1998], a large-scale plasma convection map has been extensively used at the high-latitude ionosphere. The convection electric field can also be obtained [see Chisham et al., 2007, for more details].

\subsection{The MF radar at Davis}

The Medium Frequency (MF) radar at Davis station $\left(68.6^{\circ} \mathrm{S}, 62.9^{\circ} \mathrm{E}\right)$ lies approximately $116 \mathrm{~km}$ northeast of ZHS and can be used to measure turbulent strength through velocity variances [Murphy and Vincent, 2000]. The MF radar consists of a square transmitting array (approximately $40^{\circ}$ in beam half-width at half maximum) and three cross-dipole receiving arrays. By operating in space-antenna mode at a frequency of $1.94 \mathrm{MHz}$ and using pulsed transmission with half-power full pulse widths of $30 \mu$ s, the three complex time series were analyzed using full correlation analysis to produce winds. The horizontal wind components are theoretically sampled at $2 \mathrm{~km}$ intervals between the heights of 64 $\mathrm{km}$ and $102 \mathrm{~km}$.

\section{Observation results}

A 10 hour continuous daytime observation by lidar radar was made on 14 November 2019 from 14:00 
UT to 24:00 UT (UT = MLT (Magnetic Local Time) $-1.75 \mathrm{hr}$; UT $=\mathrm{LT}-5 \mathrm{hr}$ ). The temporal and altitude variation of sodium density profile from the vertical beam, with a 5 min time and a $450 \mathrm{~m}$ altitude resolution, is shown in Figure 1a. The number of ionospheric echoes recorded by the colocated DPS-4 Digisonde in the E layer at 130 frequencies from 3 to $9.5 \mathrm{MHz}$, with $7.5 \mathrm{~min}$ time and $2.5 \mathrm{~km}$ height resolution, is shown in Figure $1 \mathrm{~b}$ as a function of virtual height and UT time simultaneously. By manually scaling the Es layer, the height variations of Es are overlaid in Figure 1a (i.e. blue asterisks). Figure 1c shows the corresponding electric field with 10 min time resolution in the northward and eastward components over ZHS derived by the SuperDARN data at Antarctica.

From Figure 1a, it can be seen that the normal sodium layer at altitudes of $\sim 85-95 \mathrm{~km}$. Starting at 17:00 UT, there is an isolated enhanced sodium density layer (i.e. the so-called SSL) observed at around $101 \mathrm{~km}$, which gradually ascends to about $103 \mathrm{~km}$ by 17:30 UT. Half an hour later, a particular region with much higher sodium density at 93-100 km until 19:15 UT can be easily identified. These regions with maximum sodium density $\sim 3.2 \times 10^{10} \mathrm{~m}^{-3}$ are about 10 times higher than the normal sodium layer. We notice that this SSL exists in two separated layers with different altitudes. One above $96 \mathrm{~km}$ with a descending trend, and the other below it with an ascending trend. After 20:00 UT, an SSL with a short time duration of about ten minutes is observed at an altitude of $97 \mathrm{~km}$.

The sodium density of the SSL observed from 17:00 to 20:20 UT changed drastically with time and altitude. This is almost consistent with the occurrence and variations of ionospheric echoes for the Es layer. The strongest SSL observed at the time interval of 18:10-19:00 UT corresponds to a large number of ionospheric echoes at almost the same height. The number of ionospheric echoes decreases at higher altitudes at the time around 17:45 and 19:05 UT, that time interval precisely corresponds to the decreased sodium density at the upper levels. Moreover, the starting and ending times of the SSLs observed from the lidar vertical beam (indicated by the gray shade in Figure 1) are aligned to the appearance and disappearance of the $E_{S}$ layer. This implies that the Es layer provides the source of sodium atoms to the SSL. With convection observations from SuperDARN radar at Antarctica, during the SSL/Es time interval, the strength of the horizontal electric field over ZHS is less than $\sim 12 \mathrm{mV} / \mathrm{m}$ (see Figure 1c), except for time at 19:20 UT, and is dominated by an eastward horizontal ion flow in the $\mathrm{F}$ region with velocities less than $170 \mathrm{~m} / \mathrm{s}$ (i.e. $\mathbf{E}=\boldsymbol{v} \times \mathbf{B}$, assuming the geomagnetic field $5.4 \times 10^{5}$ $\mathrm{nT})$. This means that the SSL/Es is located in the duskside convection cell. 
To investigate the relationship between the SSLs sodium density and ionospheric ES layer variations in more detail, Figure 2a shows variations of the SSL's in the maximum sodium density from 16:30 to 20:30 UT between 93 and $103 \mathrm{~km}$. Figure $2 \mathrm{~b}$ represents the ratio of the maximum sodium density to the background normal sodium density at the same altitude. The background normal sodium density is the averaged value between 14:30 and 16:30 UT at each altitude where the sodium density varied smoothly with time. We should notice that both sodium density in Figure $2 \mathrm{a}$ and the ratio in Figure $2 \mathrm{~b}$ are plotted with an exponential scale on the Y-axis. The critical frequency of the ES layer $\left(f_{\mathrm{o}} \mathrm{Es}\right)$ is shown in Figure 2c. The altitude of the maximum sodium density is shown in Figure 2d (blue line), while the Es layer-related virtual height variations (i.e. h'Es), with a $2.5 \mathrm{~km}$ error bar indicating possible manual scaling errors, are also overlaid.

The SSL starts at an altitude of around $102.5 \mathrm{~km}$, and the maximum sodium density increases from $\sim 1.0 \times 10^{9}$ to $\sim 3.4 \times 10^{9} \mathrm{~m}^{-3}$ within $10 \mathrm{~min}$ between $17: 02$ and 17:12 UT. Soon after, the maximum sodium density gradually decreases to $\sim 0.04 \times 10^{9} \mathrm{~m}^{-3}$ at an altitude of about 101.5 $\mathrm{km}$ till 18:07 UT, which is about twice the background sodium density. A strong SSL with double-layers occurred around 18:30 UT, and its maximum sodium density varies from $\sim 3.3 \times 10^{9}$ to $\sim 3.2 \times 10^{10} \mathrm{~m}^{-3}$, which is about twenty times higher than the background intensity. Finally, before the end of the SSL at 20:15 UT, we find the maximum sodium density is almost equal to the onset of SSLs at 17:05 UT and 18:10 UT, but with a lower height at $96.5 \mathrm{~km}$. From Figure 2c, we can see the ending of the SSL is associated with the Es layer for $f_{0}$ Es at $\sim 5 \mathrm{MHz}$. This would correspond to the ionospheric electron density at $3.1 \times 10^{11} \mathrm{~m}^{-3}$. Moreover, it can be seen that the enhancement of the Es layer at 16:55 UT and 18:00 UT occurs before the onset of the SSL by at least $5 \mathrm{~min}$. Because the Digisonde observation mode was operated at a limited frequency range (i.e. $0.5-9.5 \mathrm{MHz}$ ), the ionospheric electron density corresponding to the maximum sodium density of the SSL cannot be properly estimated over this time interval. In Figure $2 \mathrm{~d}$, we can see the variations of the SSL height (blue line) are intimately associated with the average height of the Es layers. The correlation coefficients for the SSL height within $5 \mathrm{~km} \mathrm{(29} \mathrm{points)} \mathrm{and} 2.5 \mathrm{~km}(18$ points) of the Es layer are 0.72 and 0.94 , respectively. All these observational results strongly indicate that the Es layer is most likely the source supply of sodium for the SSL in this event. However, it is difficult to explain the formation of SSL with double layers (i.e. with upper-layer and lower-layer) at 18:30-18:45 UT. 
To better understand the dynamic properties of SSL, Figures $3 a-3 c$ show height and time variations of the raw photon count, with temporal $15 \mathrm{~s}$ and spatial $45 \mathrm{~m}$ resolutions, from the (a) south, (b) vertical, and (c) west beams at 90-100 km during 18:00-19:00 UT. We have normalized the count data with the Rayleigh scattering intensity at the lower height, removing the effects of the fluctuations in the laser power and the transmittance in the lower atmosphere. The normalized raw photon count intensity would thus be in proportion to the sodium number density and the resonance scattering cross-section. The SSLs with upper-layer and lower-layer located above and below $96 \mathrm{~km}$ respectively, are observed at all three positions. Both the upper-layer and lower-layer of the SSLs are characterized by an increased photon count with a narrow altitude range (i.e. typically $\sim 1 \mathrm{~km}$ full width at half maximum). The corresponding time variations in the raw count intensity data for $92-96 \mathrm{~km}$ height are also shown in Figures $3 d-3 f$. The red, blue, and black lines represent the mean, median, and peak values of the raw count intensity. The timings (i.e. onset and ending times) of the SSL lower-layer determined at each beam, i.e. twice the background value, by stating that the ratio of the maximum density to the background normal density is greater than 2 [cf. Simonich et al., 2005], using both the mean and the median, are denoted as vertical dashed lines at each panel. For the mean values, it can be seen that the SSL lower-layer first occurred in the south beam at 18:25:42 UT. After 50 seconds, the enhanced raw photon count is observed by the vertical beam, whereas the onset time in the west beam lagged by 11 min. A similar situation with a time lag of $\sim 9.08 \mathrm{~min}$ is also observed when comparing the ending time from vertical to west positions. Moreover, through comparing the median values between the vertical and west beams, time lags of $\sim 11.82 \mathrm{~min}$ and $\sim 10.73 \mathrm{~min}$, respectively, for onset and ending time are also observed. However, the ending of the SSL lower-layer at the south beam is different. This is due to the ending of the SSL lower layer being accompanied by a wavelike perturbation (see Figure 3a). Considering the time lags for onset and end of the SSL in the raw count intensity variations at the vertical and west beams, it is suggested that the horizontal advection of enhanced sodium density plays an important role for the SSL.

\section{Discussion}

\subsection{SSL production from the Es layer}


It is important to understand the source that is producing/providing a large number of sodium atoms for

SSLs within a short timescale and a thin vertical range. In this paper, we report an SSL with spatial and temporal variations over ZHS, Antarctica. The dynamic process of the SSL is closely associated with the evolution of the Es layer, which means that the conversion of sodium ions to sodium atoms in an Es layer occurred. Previous studies have discussed the Es layer as a major source for high-density SSLs appearing between 90 and $100 \mathrm{~km}$. Kane et al. [1993] estimated that the sodium ion abundance in an Es layer was $10 \%$ of that of the atoms at most, while a $4 \%$ assumption for the sodium ion abundance was an underestimation [Hansen and von Zahn, 1990]. In our observations, the SSL occurring after 18:10 UT is at a height below $100 \mathrm{~km}$ and the SSL and Es layer have a good height correlation. An SSLrelated sodium density more than twice higher than the background intensity is observed at 18:1018:45 UT and 20:10-20:15 UT (see Figure 2b). The averaged maximum sodium density of the SSLs (using an altitude resolution of $0.45 \mathrm{~km}$ ) at these time intervals are $\sim 1.67 \times 10^{10} \mathrm{~m}^{-3}$ and $\sim 2.8 \times 10^{9} \mathrm{~m}^{-3}$, respectively. If we assume that the averaged maximum sodium density is charge exchanged from sodium ions in the Es layer at the $10 \%$ rate noted above, this should correspond to an ionospheric density of at least $\sim 1.67 \times 10^{11} \mathrm{~m}^{-3}$ and $\sim 2.8 \times 10^{10} \mathrm{~m}^{-3}$ at the same height. During these intervals, the Es layer with $f_{0}$ Es more than $5 \mathrm{MHz}$ (i.e. $\sim 3.1 \times 10^{11} \mathrm{~m}^{-3}$ ) is always observed. This means that the hypothesis about the Es layer alone providing enough of a supply of sodium atoms in this event is plausible.

Ground-based sodium lidar and ionosonde simultaneous observations of the SSL and Es layers in time and spatial location have been extensively studied by previous authors [e.g., von Zahn and Hansen, 1988; Williams et al., 2007; Dou et al., 2009]. Although the Es layer is expected to be acting as the sodium reservoir, and a strong correlation of simultaneous occurrence of SSLs and Es layers was also observed, the sodium ion chemistry seemed to not provide for a satisfactory explanation of the fast rise of sodium atom density within the growth phase of SSLs with time constants of 5 min [von Zahn and Hansen, 1988]. For our current study, the growth phase of SSLs with typical time constants of 10-15 min (see Figure 2a, i.e. the time duration from onset to the maximum of the SSL), and an electron enhancement of the Es layer preceding the sodium enhancement (see Figures 2a and 2c) is always observed, which means that our Es layer would be the source of sodium for the SSL. From a statistical point of view, a seasonal dependence of SSL occurrence correlates well with the annual variation of Es, 
as studied by Dou et al. [2010]. A "meteor-Es-SSL" chain responsible for the recombination process was taken into consideration by them. However, because meteoric ablation should simultaneously generate sodium and electron density enhancements at the same altitude [Clemesha et al., 1978], the observed preexisting Es layer with wavelike fluctuations should rule out direct meteor deposition as the cause of the SSL.

To exclude the possible redistribution of the background layer resulting in SSLs, the time-sequential relationship of the SSL/Es is investigated in more detail. Table 1 gives the estimates of the average descending velocity of the Es layer for the four-time intervals at the lower E region. Comparing these times to the SSL onset times of at $\sim 17: 02, \sim 18: 30$, and $\sim 20: 00$ UT, and with altitudes of $\sim 101, \sim 93$, and $\sim 97 \mathrm{~km}$, it seems likely that the descent of the Es layer would trigger the release of sodium atoms, while the corresponding height may be one important factor controlling the SSL occurrence and its lifetime. A theoretical explanation proposed by Cox and Plane [1998] has been shown that sodium ions can be neutralized via an ion-molecule mechanism which describes the conversion of sodium ions to atomic sodium in a descending Es layer. Similar observational events were also reported by previous studies [Beatty et al., 1989; Kane et al., 1993; Williams et al., 2007]. By identifying the vertical and temporal structures of the SSL sodium and the Es layer electron density, Kane et al. [1993] found the electron enhancement preceded the sodium enhancement at an altitude of 93-97 km were in phase. In our event, sodium enhancements always correlate with the descent of the Es layer. The high correlation between the altitude and abundance variations of the SSL and Es layers illustrates that the SSL formation is strongly related to descending Es layer and most likely involves the neutralization of sodium ions.

\subsection{SSL/Es related dynamics and electrodynamics}

Dynamic processes are evident in the temporal and spatial wavelike structures of the SSL/Es layers. With the thin Es layer declining into the E region, both the electric field and neutral wind effect on the modulation of the Es layer are expected [e.g., Nygrén et al., 1984, 2008]. According to dynamic and electrodynamic theory illustrated by Kirkwood and Nilsson [2000], the vertical motion of the ion is governed by electric fields, neutral wind, gravity, and ambipolar diffusion. Ignoring ion diffusion due to gradients in the plasma pressure and to the force of gravity, the vertical motion of ions can be expressed as: 


$$
v_{i z}=\frac{1}{1+r_{i}^{2}}\left[\frac{E_{E}}{B_{0}}+W_{N} \sin I\right] \cos I+\frac{r_{i}}{1+r_{i}^{2}}\left[-\frac{E_{N}}{B_{0}}+W_{E}\right] \cos I+\left[1-\frac{\cos ^{2} I}{1+r_{i}^{2}}\right] W_{Z}
$$

where $v_{i z}$ is the vertical motion of ion (positive downward), $r_{i}$ is the ratio of the ion-neutral collision frequency to the ion gyrofrequency. $E_{E}$ and $E_{N}$ represent the eastward and northward components of the electric field, while $W_{N}, W_{E}$, and $W_{Z}$ are the horizontal (northward and eastward) and vertical (positive downward) components of the neutral wind. The magnetic dip angle $I$ equal to $71.6^{\circ}$ at ZHS. As can be seen from the equation on the right side, the ion motion above $120-130 \mathrm{~km}$ is dominated by the first term (i.e., eastward electric field and meridional wind) due to the region ion gyro-frequency exceeds the collision frequency, while the zonal wind and north-south component of the electric field control it at heights below $100-110 \mathrm{~km}$ in the second term. Moreover, when the atmospheric gravity wave exists, the third term may be significant at any height in the presence of strong vertical motion. The present SSL/Es event mainly occurs in altitude range of $93-100 \mathrm{~km}$ with a southward electric field, and both the zonal wind and gravity waves contribute to the vertical motion of the Es layer.

The contours of the zonal and meridional wind observed by the lidar system between 14:00 and 24:00 UT are illustrated in Figures 4a and 4b, respectively. While their wind variations with height are shown in Figure 4c. The zonal wind shows a persistent westward velocity at the heights of 92.5-97 km until 20:00 UT, while a downward propagating phase structure is apparent in the wind. As studied by Kirkwood and Nilsson [2000] for the relative contributions of the electric field and neutral wind in the equation for the second term, a strong wind of $100 \mathrm{~m} / \mathrm{s}$ has almost the same effect as a rather small electric field of only $5 \mathrm{mV} / \mathrm{m}$. During the time interval of 17:00 - 20:15 UT the northward electric field with a median value of $-8.7( \pm 4.1) \mathrm{mV}$ was obtained, assuming the zonal wind with a consistent velocity of $-50 \mathrm{~m} / \mathrm{s}$ and adopting the ratio $r_{i}$ varied with the descending height [Xue et al., 2013, see their Figure 5a], the combined electric field and neutral wind will result in the Es layer with a descending speed of $\sim 7.82( \pm 6.04) \mathrm{m} / \mathrm{s}$ at $110 \mathrm{~km}$ and $\sim 0.42( \pm 0.32) \mathrm{m} / \mathrm{s}$ at $95 \mathrm{~km}$. This is as a whole consistent with the estimates of the average descending velocity of the Es layers shown in Table 1, which implies that the vertical wind velocity decreased sharply with decreasing altitude. Accumulation of metal ions and/or electrons modulated by the varied north-south electric field component and zonal wind at a certain altitude would be expected. 
layers could also be related to atmospheric gravity wave activity. MacDougall et al. [2000] suggested that such atmospheric wave motion is very efficient in moving ionization up or down, particularly at lower heights. The ionization will be concentrated near the gravity wave nulls, with a downward motion from above and upward motion from below. This process has been observed in this event at around 18:30 UT at a low height of $\sim 93 \mathrm{~km}$, where the sodium density is largest. During this interval, the SSL upper layer is descending with the downward motion of the Es layer, followed by the SSL lower layer ascending.

To assess the gravity wave activity, the $1.94 \mathrm{MHz}$ MF data from Davis station which lies approximately $116 \mathrm{~km}$ northeast of ZHS has been analyzed. To balance short-term variations against the quality of tidal fit, a 4-day window of hourly averaged winds is chosen to calculate the amplitude and time of a maximum of the diurnal, semidiurnal, and terdiurnal components of the zonal and meridional wind. These fitted parameters are then used to reconstruct the underlying tidal wind field over a two-day interval centered on the occurrence of the SSL. The MF radar wind determinations were averaged into 10 min bins over the days surrounding the ZHS SSL event. Tidal fits were made to a 4-day interval of these data and the reconstructed tides were removed from the time series [Murphy, 2002]. The resulting perturbation winds with a 3-point smoothing applied are thus shown in Figures 4d (zonal) and 4e (meridional), while the corresponding perturbation velocities at $92 \mathrm{~km}$ are shown in Figures $4 \mathrm{f}$. Downward propagating phase structures are apparent in the upper levels of these panels (i.e., Figures $4 \mathrm{~d}$ and $4 \mathrm{e}$ ) suggesting the presence of an upward propagating gravity wave. These phase structures are steep, with rapid velocity transitioning from positive to negative at the time of the SSL, indicating a large vertical wavelength node at the observed heights. The performance of the gravity wave activity illustrated by the MF radar data at the nearby station suggests such atmospheric wave motions could also modulate the temporal and spatial variations of the SSL intensities.

In comparison to 5 min averaged data shown in Figure 1a, there is another interesting feature that the $15 \mathrm{~s}$ data in Figure 3 reveals considerably more fine structures of the SSL. In Figures $3 \mathrm{a}-3 \mathrm{c}$, the double layers of the SSL show predominant wavelike structures with periods of 7-11 min at 96-100 km and $\sim 3-4$ min at 94-96 km, which is especially evident in the south direction in Figure 3a. This is very different from the observations of the Es layer at ionosonde and the gravity wave at MF radar, in which only a long period of perturbations could be derived. Quasi-periodic fluctuations on a timescale on the 
order of several minutes in the peak height and the peak density of SSLs as a universal feature but could be concealed by the lower temporal resolution [Pfrommer et al., 2009]. Tsuda et al. [2011] suggested that the 7-11 min wavelike structure would be signatures of atmospheric gravity waves, while the 3 min wavelike structure may be signatures of an atmospheric gravity wave or acoustic wave. Moreover, Hansen and von Zahn [1990] analyzed sodium density data with time-resolution of 1-min and demonstrated upward and downward movements of the SSL height with the time scale of $~ 20$-min. They also suggested that such movements are signatures of atmospheric gravity waves. However, the careful reader will notice that the fine-scale $\sim 3$ min wavelike structure seems to occur locally (see Figure 3a), due to the vertical and west beams without detecting such fine-scale wavelike structure at the lower layer of SSL. In conclusion, as the lidar probes the sodium along with three directions, variations will occur as the SSL is carried across the beam by the neutral wind. The changes that we see could be some combination of intrinsic temporal changes and spatial variations along the direction of advection.

As mentioned in the Introduction, horizontal advection of the SSLs was hypothesized by Clemesha et al. [1980], but no background wind confirms it. By detecting the onset time and amounts of the sodium density of SSLs via a five-direction lidar system combining derived background wind, Tsuda et al. [2015] verify the horizontal advection of the SSLs. However, the ending time of their SSLs was not available due to the cloudy sky. Since the sodium lidar observed SSLs in the vertical, and $30^{\circ}$ off-zenith to the south and west, it is possible to assess the role of advection in SSL variation. From Figures 3e and 3f, we observe the SSL lower layer has similar structures in the vertical and west beams, while the lifetime of the SSL is about $14.63 \mathrm{~min}$ at the vertical position and $12.65 \mathrm{~min}$ at the west position, respectively. The onset and ending time lags between the vertical and west beams are about $11 \mathrm{~min}$ and $9.1 \mathrm{~min}$, respectively. Cross-correlation analysis of the SSL upper-layer and lower-layer also indicates that both layers with a time lag of about 11 min between the vertical and west beams. The height of SSL at $94 \mathrm{~km}$ corresponds to the distance of about $54.27 \mathrm{~km}$ between the vertical and west beams. Assuming the time lags of the SSL in the vertical and west beams are due to horizontal advection, the bulk velocity of the SSL onset is estimated to be $82.2( \pm 1.8) \mathrm{m} / \mathrm{s}$ and its rear is estimated to be $99.4( \pm 1.8) \mathrm{m} / \mathrm{s}$ westward. These velocities are fairly consistent with the observed zonal wind velocity derived from the lidar system shown in Figure $4 \mathrm{c}(\sim 80-90 \mathrm{~m} / \mathrm{s}$ westward $)$. This suggests that 
the temporal and spatial variations of the SSL are consistent with advection by the background wind.

\section{Summary}

398 A new sodium resonance fluorescence Doppler lidar system with three-directional beams has been established at ZHS, Antarctica. In this paper, we report an SSL with spatial and temporal variations on 14 November 2019. By examining the dynamic properties of this SSL, and comparing it with collocated Es layers, we find the dynamic process of the SSL is closely associated with the evolution of the Es layer. We suggest the formation and perturbation of the SSL correlates with the convective electric field and atmospheric gravity wave activity. However, the onset/end of the SSL was observed by lidar at different times in the different beams, especially in the east-west direction. By using observational atmospheric wind field values obtained by the lidar system itself, we have compared wind velocities to the calculated horizontal advection effect and found the velocities are consistent. We conclude that the major source for sodium atoms in this SSL event is from the Es layer and the dynamic properties of the SSL are modulated by the Es layer electrodynamics and the background wind field.

\section{Data Availability Statement}

412 The ZHS DPS and lidar data can be downloaded from the polar atmospheric and space physics database in Chinese National Arctic and Antarctic Data Center (http://www.chinare.org.cn:8000/uap/).

414 The HF radar data used in this work is available from the Virginia Tech portal at http://vt.superdarn.org.

415 Davis MF radar data is available from the Australian Antarctic Data Centre at https://data.aad.gov.au.

\section{Acknowledgments}

This work is supported by the National Key Research and Development Program of China (Grant numbers 2016YFC1400300, 2021YFE0106400, and 2018YFC1407300), the National Natural Science Foundation of China (Grant numbers 41774166, 41704159, 41831072), the International Cooperation

421 Advance Research on Key Scientific Issues of the International Meridian Project (A131901W14), and the fund from Institute of Applied Meteorology. The author also acknowledges the use of data from the Chinese Meridian Project. SuperDARN is a collection of scientific HF radars funded by the national 
424 science funding agencies of Australia, Canada, China, France, Italy, Japan, Norway, South Africa, the 425 United Kingdom, and the United States of America. The operation of the Davis MF radar is supported 426 under the Australian Antarctic Science project 4445.

427 


\section{References}

Batista, P. P., B. R. Clemesha, and D. M. Simonich (1991), Horizontal structures in sporadic sodium layers at $23^{\circ} \mathrm{S}$, Geophysical Research Letters, 18(6), 1027-1030, doi:10.1029/91GL00549.

Beatty, T. J., R. L. Collins, C. S. Gardner, C. A. Hostetler, C. F. Sechrist Jr., and C. A. Tepley (1989), Simultaneous radar and lidar observations of sporadic $\mathrm{E}$ and $\mathrm{Na}$ layers at Arecibo, Geophysical Research Letters, 16(9), 1019-1022, doi:https://doi.org/10.1029/GL016i009p01019.

Bedey, D. F., and B. J. Watkins (1997), Large-scale transport of metallic ions and the occurrence of thin ion layers in the polar ionosphere, Journal of Geophysical Research Space Physics, 102(A5), 9675-9681, doi: 10.1029/96ja03825.

Chisham, G., et al. (2007), A decade of the Super Dual Auroral Radar Network (SuperDARN): scientific achievements, new techniques and future directions, Surveys in Geophysics, 28(1), 33-109, doi:10.1007/s10712-007-9017-8.

Chu, X., and G. Papen (2005), Resonance Fluorescence Lidar for Measurements of the Middle and Upper Atmosphere, in Laser Remote Sensing, edited by Fujii, T. and Fukuchi, T., pp. 179-432, CRC Press, Boca Raton, Fla.

Clemesha, B. R. (1995), Sporadic neutral metal layers in the mesosphere and lower thermosphere, Journal of Atmospheric and Terrestrial Physics, 57(7), 725-736, doi:10.1016/0021-9169(94)00049-T.

Clemesha, B. R., V. W. J. H. Kirchhoff, D. M. Simonich, and H. Takahashi (1978), Evidence of an extra-terrestrial source for the mesospheric sodium layer, Geophysical Research Letters, 5(10), 873-876, doi:10.1029/GL005i010p00873.

Clemesha, B. R., V. W. J. H. Kirchhoff, D. M. Simonich, H. Takahashi, and P. P. Batista (1980), Spaced lidar and nightglow observations of an atmospheric sodium enhancement, Journal of Geophysical Research: Space Physics, 85(A7), 3480-3484, doi:10.1029/JA085iA07p03480.

Collins, S. C., et al. (2002), A study of the role of ion-molecule chemistry in the formation of sporadic sodium layers, Journal of Atmospheric and Solar-Terrestrial Physics, 64(7), 845-860, doi:10.1016/S1364-6826(02)00129-3.

Cox, R. M., and J. M. C. Plane (1998), An ion-molecule mechanism for the formation of neutral sporadic Na layers, Journal of Geophysical Research: Atmospheres, 103(D6), 6349-6359, doi:10.1029/97jd03376.

Croskey, C. L., C. N. Mitchell, M. Friedrich, F. J. Schmidlin, and R. A. Goldberg (2006), Sporadic sodium and E layers observed during the summer 2002 MaCWAVE/MIDAS rocket campaign, Annales Geophysicae, 24, 1257-1266, doi:10.5194/angeo-24-1257-2006.

Dou, X. K., X. H. Xue, T.-D. Chen, W.-X. Wan, and Z.-Y. Chen (2009), A statistical study of sporadic sodium layer observed by Sodium lidar at Hefei (31.8 ${ }^{\circ}$, 117.3 E), Annales Geophysicae, 27(6), 2247-2257, doi:10.5194/angeo-27-2247-2009.

Dou, X. K., X. H. Xue, T. Li, T. D. Chen, C. Chen, and S. C. Qiu (2010), Possible relations between meteors, enhanced electron density layers, and sporadic sodium layers, Journal of Geophysical Research: Space Physics, 115(A6), A06311, doi:10.1029/2009ja014575.

Grebowsky, J. M., and A. C. Aikin (2001), In Situ Measurements of Meteoric Ions, 189-214 pp., Cambridge Univ. Press, Cambridge, U. K., edited by E. Murat and I. P. Williams.

Greenwald, R. A., et al. (1995), DARN/SuperDARN A global view of the dynamics of high-latitude convection, Space Science Reviews, 71(1-4), 761-796, doi:10.1007/BF00751350.

Hansen, G., and U. von Zahn (1990), Sudden sodium layers in polar latitudes, Journal of Atmospheric and Terrestrial Physics, 52(6), 585-608, doi:10.1016/0021-9169(90)90055-R.

Heinselman, C. J., J. P. Thayer, and B. J. Watkins (1998), A high-latitude observation of sporadic sodium and sporadic E-layer 
formation, Geophysical Research Letters, 25(16), 3059-3062, doi:10.1029/98g102215.

Kane, T. J., C. S. Gardner, Q. Znou, J. D. Mathews, and C. A. Tepley (1993), Lidar, radar and airglow observations of a prominent sporadic Na/sporadic E layer event at Arecibo during AIDA-89, Journal of Atmospheric and Terrestrial Physics, 55(3), 499-511, doi:10.1016/0021-9169(93)90084-C.

Kirkwood, S., and P. N. Collis (1989), Gravity wave generation of simultaneous auroral sporadic-E layers and sudden neutral sodium layers, Journal of Atmospheric \& Terrestrial Physics, 51(4), 259,263-261,269, doi:10.1016/0021-9169(89)90077-9.

Kirkwood, S., and U. V. Zahn (1991), On the role of auroral electric fields in the formation of low altitude sporadic-E and sudden sodium layers, Journal of Atmospheric \& Terrestrial Physics, 53(5), 389-407, doi:10.1016/0021-9169(91)90034-5.

Kirkwood, S., and H. Nilsson (2000), High-latitude Sporadic-E and other Thin Layers - the Role of Magnetospheric Electric Fields, Space Science Reviews, 91(3), 579-613, doi:10.1023/A:1005241931650.

Kopp, E. (1997), On the abundance of metal ions in the lower ionosphere, Journal of Geophysical Research: Space Physics, 102(A5), 9667-9674, doi:10.1029/97ja00384.

MacDougall, J. W., and P. T. Jayachandran (2005), Sporadic E at cusp latitudes, Journal of Atmospheric and Solar-Terrestrial Physics, 67(15), 1419-1426, doi:10.1016/j.jastp.2005.07.011.

MacDougall, J. W., P. T. Jayachandran, and J. M. C. Plane (2000), Polar cap Sporadic-E: Part 1, Observations, Journal of Atmospheric and Solar-Terrestrial Physics, 62(13), 1155-1167, doi:10.1016/S1364-6826(00)00093-6.

Milan, S. E., T. K. Yeoman, M. Lester, E. C. Thomas, and T. B. Jones (1997), Initial backscatter occurrence statistics from the CUTLASS HF radars, Annales Geophysicae, 15(6), 703-718, doi:10.1007/s00585-997-0703-0.

Murphy, D. J., and R. A. Vincent (2000), Amplitude enhancements in Antarctic MF radar echoes, Journal of Geophysical Research: Atmospheres, 105(D21), 26683-26693, doi:10.1029/2000jd900510.

Murphy, D. J. (2002), Variations in the phase of the semidiurnal tide over Davis, Antarctica, Journal of Atmospheric and Solar-Terrestrial Physics, 64, 1069-1081.

Nygrén, T., L. Jalonen, J. Oksman, and T. Turunen (1984), The role of electric field and neutral wind direction in the formation of sporadic E-layers, Journal of Atmospheric and Terrestrial Physics, 46(4), 373-381, doi:10.1016/0021-9169(84)90122-3.

Nygrén, T., M. Voiculescu, and A. T. Aikio (2008), The role of electric field and neutral wind in the generation of polar cap sporadic E, Annales Geophysicae, 26, 3757-3763, doi:10.5194/angeo-26-3757-2008.

Pfrommer, T., P. Hickson, and C.-Y. She (2009), A large-aperture sodium fluorescence lidar with very high resolution for mesopause dynamics and adaptive optics studies, Geophysical Research Letters, 36(15), n/a-n/a, doi:10.1029/2009g1038802.

Qiu, S., W. Soon, X. Xue, T. Li, W. Wang, M. Jia, C. Ban, X. Fang, Y. Tang, and X. Dou (2018), Sudden Sodium Layers: Their Appearance and Disappearance, Journal of Geophysical Research: Space Physics, 123(6), 5102-5118, doi:10.1029/2017ja024883.

Qiu, S., Y. Tang, and X. Dou (2015), Temperature controlled icy dust reservoir of sodium: A possible mechanism for the formation of sporadic sodium layers, Advances in Space Research, 55(11), 2543-2565, doi:10.1016/j.asr.2015.02.011.

Qiu, S., Y. Tang, M. Jia, X. Xue, X. Dou, T. Li, and Y. Wang (2016), A review of latitudinal characteristics of sporadic sodium layers, including new results from the Chinese Meridian Project, Earth-Science Reviews, 162, 83-106, doi:10.1016/j.earscirev.2016.07.004.

Reid, I. M., D. L. McIntosh, D. J. Murphy, and R. A. Vincent (2018), Mesospheric radar wind comparisons at high and middle southern latitudes, Earth, Planets and Space, 70(1), doi:10.1186/s40623-018-0861-1. 
Reinisch, B. W., et al. (2008), Advancing Digisonde Technology: the DPS-4D, in AIP Conference Proceedings, edited, pp. 127-143, doi:10.1063/1.2885022.

Reinisch, B. W., I. A. Galkin, G. M. Khmyrov, A. V. Kozlov, K. Bibl, I. A. Lisysyan, G. P. Cheney, X. Huang, D. F. Kitrosser, and V. V. Paznukhov (2009), New Digisonde for research and monitoring applications, Radio Science, 44(1), 1-15, doi:10.1029/2008RS004115.

Ruohoniemi, J. M., and K. B. Baker (1998), Large-scale imaging of high-latitude convection with Super Dual Auroral Radar Network HF radar observations, Journal of Geophysical Research, 103(A9), 20797, doi:10.1029/98ja01288.

Simonich, D., B. Clemesha, and P. P. Batista (2005), Sporadic sodium layers and the average vertical distribution of atmospheric sodium, Advances in Space Research, 35(11), 1976-1980, doi:10.1016/j.asr.2005.06.030.

Takahashi, T., et al. (2015), A case study on generation mechanisms of a sporadic sodium layer above Tromsø $\left(69.6^{\circ} \mathrm{N}\right)$ during a night of high auroral activity, Annales Geophysicae, 33(8), 941-953, doi:10.5194/angeo-33-941-2015.

Thomas, L., A. J. Gibson, and S. K. Bhattacharyya (1977), Lidar observations of a horizontal variation in the atmospheric sodium layer, Journal of Atmospheric \& Terrestrial Physics, 39(11-12), 1405-1409, doi:10.1016/0021-9169(77)90095-2.

Tsuda, T. T., et al. (2011), Fine structure of sporadic sodium layer observed with a sodium lidar at Tromsø, Norway, Geophysical Research Letters, 38(18), L18102, doi:10.1029/2011g1048685.

Tsuda, T. T., et al. (2015), A sporadic sodium layer event detected with five-directional lidar and simultaneous wind, electron density, and electric field observation at Tromsø, Norway, Geophysical Research Letters, 42(21), 9190-9196, doi:10.1002/2015gl066411.

Von Zahn, U., and T. L. Hansen (1988), Sudden neutral sodium layers: a strong link to sporadic E layers, Journal of Atmospheric and Terrestrial Physics, 50(2), 93-104, doi:https://doi.org/10.1016/0021-9169(88)90047-5.

Williams, B. P., F. T. Berkey, J. Sherman, and C. Y. She (2007), Coincident extremely large sporadic sodium and sporadic E layers observed in the lower thermosphere over Colorado and Utah, Annales Geophysicae, 25(1), doi:10.5194/angeo-25-3-2007.

Xue, X. H., X. K. Dou, J. Lei, J. S. Chen, Z. H. Ding, T. Li, Q. Gao, W. W. Tang, X. W. Cheng, and K. Wei (2013), Lower thermospheric-enhanced sodium layers observed at low latitude and possible formation: Case studies, Journal of Geophysical Research: Space Physics, 118(5), 2409-2418, doi:10.1002/jgra.50200. 
Figure 1: (a) Height-time intensity variations in the sodium density from 14:00 to 24:00 UT on 14 November 2019 between 80 and $105 \mathrm{~km}$ are presented. Blue asterisks denote the height of the Es layer derived from DPS-4 Digisonde. (b) The number of ionospheric echoes recorded by the colocated DPS-4Digisonde in the E layer at frequencies from 3 to $9.5 \mathrm{MHz}$. (c) The corresponding electric field derived from SuperDARN data, the blue and red lines represent eastward and northward components, respectively. In this study, we mainly focus on the SSL occurring for time intervals shown in the gray shade (i.e. 17:00-20:20 UT)

Figure 2: (a) Variations of the SSL's maximum sodium density from 16:30 to 20:30 UT. (b) The ratio of the maximum sodium density to the background sodium density at the same altitude. Horizontal black dash line denotes values with a ratio greater than 2. (c) The variations of critical frequency in Es layer from colocated DPS-4 Digisonde. (d) Variations in altitude of the maximum SSL (blue line) and the Es layer (red asterisks with error bar).

Figure 3: (a-c) Height and time variations of raw photon count, with $15 \mathrm{~s}$ time resolution and $45 \mathrm{~m}$ height resolution, from (a) south, (b) vertical, and (c) west beams. (d-f) The corresponding time variations in the raw count intensity data for 92-96 km height. Red, blue, and black lines represent data obtained with the mean, median, and peak values, respectively. (notice: the peak values have been reduced by a factor of three). The onset and end times, defined as a time when the intensity became twice that before the event, are shown by vertical dash lines.

Figure 4: Time-height contours of the zonal (a) and meridional (b) winds between 14:00 and 24:00 UT derived from sodium radar. The data has been smoothed to have a vertical resolution of $0.5 \mathrm{~km}$ and a temporal resolution of 1 hour for the wind field. (c) Profiles of zonal (red and blue) and meridional (black and green) winds at 17:3018:30 and 18:30-19:30 UT. Zonal (d) and meridional (e) 10 min average winds from the Davis MF radar for two days around the ZHS SSL occurrence. The tidal variations have been removed with the gravity wave activity remaining. (f) Zonal (red) and meridional (black) perturbation winds at $92 \mathrm{~km}$.

Table 1: Estimates of average descending velocity of the Es layers

\begin{tabular}{rccc}
\hline Time interval (hh:mm:ss) & Initial height $(\mathrm{km})$ & Ending height $(\mathrm{km})$ & Descending vel. $(\mathrm{m} / \mathrm{s})$ \\
\hline $16: 45: 10-17: 30: 10$ & 120 & 100 & $\sim 7.41$ \\
$17: 37: 40-18: 30: 10$ & 122.5 & 92.5 & $\sim 9.52$ \\
$19: 00: 10-19: 30: 10$ & 122.5 & 97.5 & $\sim 13.89$ \\
$19: 52: 40-20: 15: 10$ & 102.5 & 97.5 & $\sim 3.7$ \\
\hline
\end{tabular}

